\title{
RECURRENT INFILTRATING DUCTAL CARCINOMA IN LEFT MASTECTOMY PLASTRON DURING PREGNANCY: A CASE REPORT
}

Juber Mateus Ellwanger ${ }^{1}$, Caio Bertolini, Samuel Cavalcante Reis¹, Daniela Takito¹, Priscila Ribas

${ }^{1}$ Universidade Federal de Pelotas - Pelotas (RS), Brazil.

Introduction: Breast cancer has the highest incidence, prevalence, and mortality rate among malignant neoplasms in women worldwide (excluding non-melanoma skin cancer). Although there are well-defined treatments, they are still controversial during pregnancy: surgery seems safe and chemotherapy (CT) poses no harm to the fetus, especially if applied late in pregnancy. Hormone therapy (HT) and radiation therapy (RT) are prone to cause fetal damage. In patients diagnosed with pregnancy during treatment, there are no clear procedures about terminating the pregnancy or ceasing CT and RT. In these cases, it is necessary to monitor the patient and the fetus taking into account the woman's will - especially if the tumor has estrogen and progesterone receptors, increasing the chance of relapsing or stop responding to CT. This study reports a case in which the hormones of pregnancy influenced a major recurrence of breast cancer, which diminished shortly after the birth. Case report: A 35-year-old woman, diagnosed with infiltrating ductal carcinoma in the left breast, underwent sectorectomy, axillary lymph nodes excision, and RT with an insufficient response. Subsequently, left tumor recurrence arose and mastectomy was performed. In the follow-up, she underwent CT and RT, with poor response. In the interim, it was discovered that the patient was pregnant, thus referred from oncology to gynecology for the interruption, since there was a considerable recurrence in the left breast plastron. Sixth -times pregnant, with five vaginal deliveries, the latest one six years before, all pregancies without complications. She was advised to terminate pregnancy but remained adamant in maintaining the pregnancy. She underwent an obstetric ultrasound showing a viable fetus of six weeks and six days of gestational age (GA). At 22 weeks of pregnancy, she was referred to the hospital by the oncologist for the interruption, as the plastron on the left breast was growing, with CT failure. The patient acknowledged that, with this GA, the fetus's chance of survival was low. Yet, she opted for pregnancy continuation. Later she was sent by the prenatal care to the maternity hospital at 32 weeks of GA, aiming at delivery and a new CT protocol afterwards. She started corticosteroids for pulmonary development of the conceptus and endured cesarean delivery with bilateral adnexectomy. Female newborn, 1.830g, 8/9 APGAR score and 32 weeks and 5 days Capurro, transferred to the neonatal ICU (intensive care unit) due to prematurity. The patient was evaluated few months after delivery: great spontaneous resolution of the plastron in the left breast, with no effect of pregnancy hormones and responsive to CT. Follow-up in the oncology department. 